\title{
Measurement-based auralization methodology for the assessment of noise mitigation measures
}

\author{
Pieter Thomas*, Weigang Wei, Timothy Van Renterghem, Dick Botteldooren \\ Department of Information Technology (INTEC), Ghent University, Technologiepark-Zwijnaarde 15, B-9052 Gent, Belgium
}

\begin{abstract}
The effect of noise mitigation measures is generally expressed by noise levels only, neglecting the listener's perception. In this study, an auralization methodology is proposed that enables an auditive preview of noise abatement measures for road traffic noise, based on the direction dependent attenuation of a priori recordings made with a dedicated 32channel spherical microphone array. This measurement-based auralization has the advantage that all non road traffic sounds that create the listening context are present. The potential of this auralization methodology is evaluated through the assessment of the effect of an L-shaped mound. The angular insertion loss of the mound is estimated by using the ISO 9613-2 propagation model, the Pierce barrier diffraction model and the Harmonoise point-to-point model. The realism of the auralization technique is evaluated by listening tests, indicating that listeners had great difficulty in differentiating between a posteriori recordings and auralized samples, which shows the validity of the followed approaches.
\end{abstract}

Keywords: measurement-based auralization, HRTF pattern emulation, noise mitigation assessment PACS: 43.50.Gf, 43.50.Rq, 43.60.Fg, 43.66.Pn

\section{Introduction}

The effect of traffic noise mitigation is generally expressed as a reduction in equivalent A-weighted level. Nonexperts such as local inhabitants, traffic planners or urban planners cannot easily transfer this single number estimate to a perceived improvement of the sonic environment. Moreover, with the more subtle design of soundscapes in mind, one should consider potential unmasking of wanted sounds as well as changes in spectrum and temporal structure. Such a design for quality would benefit from auralizing the expected effect much like product manufacturers use auralizations for product sound quality design.

Existing approaches are commonly based on ab initio auralization techniques. Maillard et al. [1,2] developed an auralization tool to model car passages in an urban environment based on a traffic flow simulator, a real time granular source synthesis technique and a moving source renderer. Individual source signals are generated by using an overlapadd mechanism on sound samples extracted from engine and rolling noise recordings, with the speed and acceleration from the traffic flow simulator as input [3]. The LISTEN-project [4,5] is another example of a research project aiming at the auralization of urban soundscapes. Here, a single car pass-by has been auralized based on individual engine and rolling noise signals, extracted from an original pass-by recording by inversion of the propagation effects.

The perceived realism of these ab initio auralizations is typically relying on the accurateness of the description of each individual vehicle noise source, depending on the local road surface, vehicle fleet composition and traffic flow.

In this paper, a new approach for the auralization of noise mitigation measures is suggested based on a priori recordings of the total sound environment. In this way, specific local effects related to the traffic such as typical traffic flow and vehicle fleet composition are readily included. Furthermore, the effect of the noise mitigation measure on the complete sound environment can be evaluated, as other sources, typical for that site, remain present and can become

\footnotetext{
${ }^{*}$ Corresponding author

Email address: pieter.thomas@intec.ugent.be (Pieter Thomas)
} 
more audible. The influence of the noise mitigation on the local soundscape experience is emulated rather than the reduction of the noise level alone.

The implementation of the proposed methodology used in this paper is based on a priori array recordings with a 32channel spherical microphone array, specifically designed to facilitate direction-based sound suppression and headrelated transfer function (HRTF) directivity pattern emulation. The effect of a noise mitigation measure is included by spatial-selective attenuation of the array directivity pattern, by only attenuating the sound coming from a certain direction. While the time-selective auralization technique, previously developed by Thomas et al. [6], could only account for a single car passage and noise mitigation measures with simple geometry, the technique presented here is very suitable for the auralization of complex-shaped noise mitigation measures in real sound environments, containing multiple sources at different locations.

The paper consists of two main parts. In a first part, the proposed auralization methodology is described in detail (Section 2). In a second part of this work, the methodology is applied to a complex L-shaped mound (Section 3). Section 3.1 describes the different simulation techniques to estimate the direction dependent attenuation of the mound. An evaluation of the accuracy of the directivity pattern emulation in terms of relative mean square error (rMSE) and white noise gain (WNG) is made in Section 3.2. In Section 3.3, a listening test is used to assess whether the proposed methodology fulfills the requirements of a tool for predicting the impact of a noise mitigation measure: (1) the effect of the measure should be clearly noticeable by a listener; (2) the listener should not be able to distinguish between the auralization and an a posteriori recording. Both are assessed in a forced choice experiment.

\section{Auralization methodology for noise mitigation measures}

The auralization technique is based on direction dependent attenuation of a priori recordings of the sound environment. If direction dependent attenuation needs to be applied for auralization purposes, information about the spatiality of the sound environment needs to be known in order to attenuate each source appropriately. A methodology to extract the direction of a single sound source based on the auditory cues of binaural recordings has been developed earlier [6], but this method proves to be insufficient when localizing multiple sound sources in a complex sound environment. To increase the degree of spatial information that can be extracted, compared to a 2-channel binaural system, a more advanced recording technique is proposed. A 32-channel spherical microphone array is optimized for the simulation of a virtual microphone with directional characteristics that approximate the directivity of the human hearing with a higher degree than the first order spherical head model, proposed by Algazi et al. [7], while improving the spatial resolution which a sound environment can be captured with. A set of weights is calculated for each microphone of the constituting array, so that the resulting array directivity pattern corresponds with the HRTF directivity pattern for the left or right ear for a given looking direction, attenuated with the direction dependent angular insertion loss (IL) of the noise mitigation measure to include source direction dependent attenuation. Figure 1 pictures the main operating principle of the array-based auralization technique. The set of optimal weights is frequency dependent, but will be restricted towards the high frequency range as directional characteristics, described by the HRTFs, become highly sensitive to spectral fluctuations due to individual differences of the pinnae. At high frequencies, only the signal from the microphone closest to the virtual ear location will be selected, corresponding to a first order spherical head approximation [7].

\subsection{Microphone array design}

The microphone array consists of a hard plastic sphere with a $7-\mathrm{cm}$ radius as a substitute for the human head, facilitating the calculation of the microphone filters needed to approximate the HRTFs. The chosen radius is somewhat smaller than the average radius of $8.7 \mathrm{~cm}$ that Algazi et al. observed [8]. In this way, the frequency at which spatial aliasing starts can be increased, while the array still allows for 2-channel spherical head approximation (cf. [7]). 32 omnidirectional microphones are distributed on the sphere allowing focus on azimuthal rather than elevational separation, corresponding to the larger sensitivity of humans regarding azimuthal source discriminating resolution [9, 10]. Apart from the two poles, the remaining 30 microphones are uniformly distributed along three circles at elevations of $-45^{\circ}, 0^{\circ}$ and $45^{\circ}$. The microphone array is depicted in Fig. 2, with the microphone positions indicated.

Knowles Acoustics type FG-23329 microphones are used in the array. These are low cost microphones (about $€ 30$ per piece), carefully selected after long-term outdoor comparison with several other low cost microphone capsules [11]. For the data-acquisition (DAQ), a National Instruments PXIe-1082 chassis with three NI-4498 DAQ cards is used. 


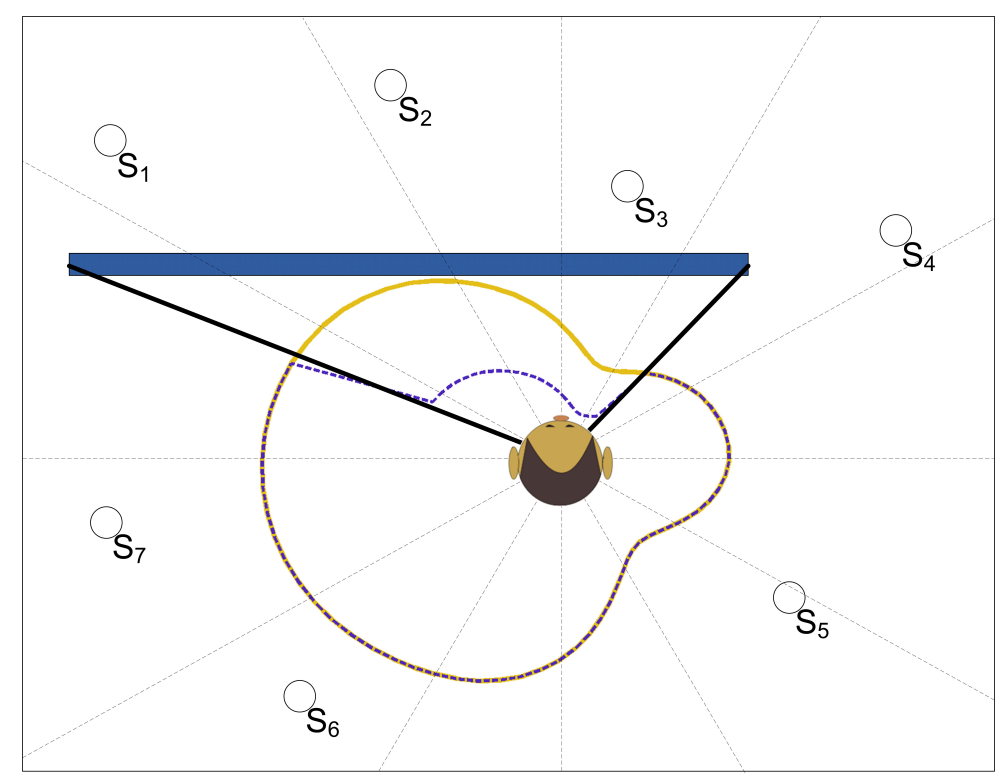

Figure 1: Operating principle of the noise mitigation measure auralization technique. The effect of a noise mitigation measure (rectangle) is introduced by spatially attenuating the HRTF directivity pattern (depicted for the left ear, full line) with the angular IL of the measure. The resulting attenuated HRTF directivity pattern (dashed line) is emulated by a 32-channel microphone array. Only sources $S_{1}-S_{3}$ behind the measure are attenuated, other sources are unaffected.

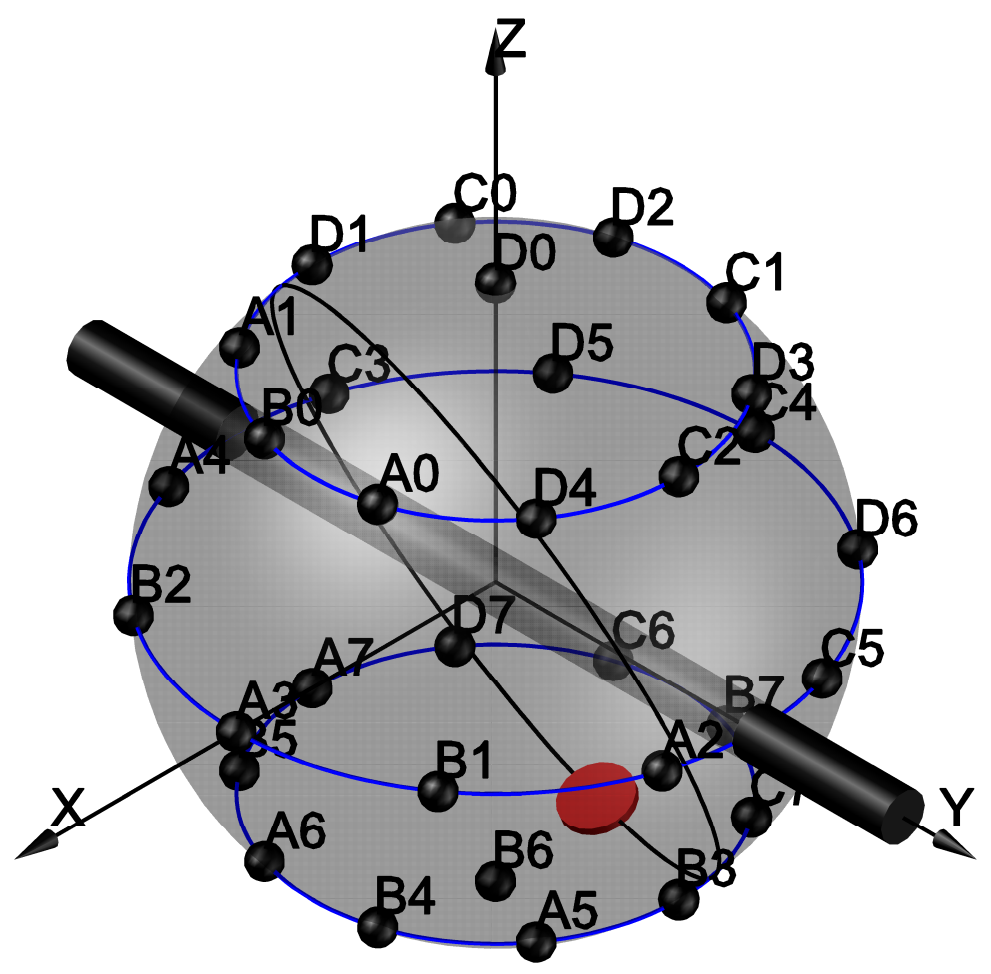

Figure 2: Spherical microphone array geometry. The positions of the 32 microphones are indicated. The bar along the y-axis is part of the fixation mechanism. The circular disk at the bottom of the sphere indicates the outlet of microphone cables. 


\subsection{Auralization based on directivity pattern emulation}

This section discusses how to determine the microphone weights so that the resulting microphone array directivity pattern emulates the HRTF directivity pattern, spatially attenuated with the angular IL of the noise mitigation measure.

\subsubsection{Characterization of the target array directivity pattern}

In a first step, the target directivity pattern has to be defined. To account for the binaural aspect, this directivity pattern is based on the frequency dependent $\operatorname{HRTF}$ directivity patterns, $\operatorname{HRTF}(f, \phi, \theta)$. The set of target $\operatorname{HRTFs}$ used here is taken from measurements by Gardner at MIT [12]. Compensation filters have been designed to account for the frequency response of the measurement equipment, ear canal and reproduction headphones used in the listening test (Sennheiser HD-280 pro, see Section 3.3).

In order to account for the effect of the noise mitigation measure, each of the impulse responses $\operatorname{HRIR}\left(t, \phi_{l}, \theta_{l}\right)$, corresponding with the equalized HRTFs for direction $\left(\phi_{l}, \theta_{l}\right)$, are decomposed into 1/3-octave band components $\operatorname{HRIR}_{i}\left(t, \phi_{l}, \theta_{l}\right)$ and attenuated with the corresponding $1 / 3$-octave band angular $\operatorname{IL}_{i}\left(\phi_{l}, \theta_{l}\right)$ of the noise mitigation measure:

$$
\operatorname{attHRIR}\left(t, \phi_{l}, \theta_{l}\right)=\sum_{i} \operatorname{HRIR}_{i}\left(t, \phi_{l}, \theta_{l}\right) 10^{-\mathrm{IL}_{i}\left(\phi_{l}, \theta_{l}\right) / 20},
$$

where attHRIR $\left(t, \phi_{l}, \theta_{l}\right)$ is the attenuated impulse response and $i$ the index of the $1 / 3$-octave band.

The set of attenuated HRTFs is only available for 710 discrete directions $\left(\phi_{l}, \theta_{l}\right)$. Therefore, an interpolation strategy needs to be developed to estimate the target directivity pattern at intermediate orientations. Furthermore, no HRTF data is known at elevations lower than $-40^{\circ}$. This implies that the target pattern needs to be extrapolated to avoid pattern blow-up when calculating optimal microphone array weights later on.

Different strategies have been developed to perform HRTF interpolation [13, 14, 15, 16, 17, 18, 19, 20, 21]. In this work, a spherical harmonic decomposition of the attenuated magnitude and unwrapped phase HRTF data is chosen, allowing a representation in terms of a set of continuous predefined orthonormal basis functions [13, 15].

Following Pollow et al. [15], the $N^{\text {th }}$-order spherical harmonic decomposition of either the attenuated HRTF magnitude or unwrapped phase is written as:

$$
\operatorname{attHRTF}_{\|, \angle}(f, \phi, \theta)=\sum_{n=0}^{N} \sum_{m=-n}^{n} A_{\|,,<}^{n m}(f) Y_{n}^{m}(\phi, \theta),
$$

where $Y_{n}^{m}(\phi, \theta)$ represents the spherical harmonic basis functions of order $\mathrm{n}$ and degree $\mathrm{m}$ and $A_{\|, \angle}^{n m}(f)$ the frequency dependent spherical harmonic coefficients for either magnitude (subscript $\|$ ) or unwrapped phase (subscript $\angle$ ), given in matrix form by $[15,22]$ :

$$
\mathbf{A}_{\|, \angle}(f)=\left[\mathbf{Y}^{H} \mathbf{V Y}+\lambda \mathbf{D}\right]^{-1} \mathbf{Y}^{H} \mathbf{V} \operatorname{attHRTF}_{\|, \angle}(f),
$$

with attHRTF $\mathbf{F}_{\|, \angle}(f)$ being the $L \times 1$ magnitude or unwrapped phase of the attenuated HRTF dataset (with $L$ representing the number of discrete orientations $\left(\phi_{l}, \theta_{l}\right)$ of the sampling grid) and $\mathbf{Y}$ the $L \times(N+1)^{2}$ matrix with spherical harmonic basis functions. $\mathbf{V}$ is an $L \times L$ diagonal matrix, encompassing a basic quadrature weighting to account for the $L$-point evaluation grid discretization when using a non-optimized open grid and to maintain the orthonormality relation of the discretized spherical harmonics. Weighting coefficients are derived from the Voronoi surfaces [23]. To further account for the open irregular grid, Tikhonov regularization is applied [24]. Here, $\lambda$ is the regularization parameter and $\mathbf{D}$ is a $(N+1)^{2} \times(N+1)^{2}$ diagonal matrix, with diagonal elements $1+n(n+1)$ to decrease the influence of higher order harmonics $[15,25]$.

The use of the least-squares methodology can cope with the polar gap when regularization is applied. However, the presence of higher order coefficients, resulting from the regularized fit on the known data, gives rise to meaningless (large) pattern fluctuations in the polar gap. In order to have more control on the data and to guarantee that the behavior of the directivity pattern is smooth in the polar gap, the spherical harmonic coefficients are calculated from a combination of the known data at available directions and the extrapolated data at the polar gap, resulting from a 
lower order $(N=3)$ approximation [26]. Additionally, the magnitude value at the south pole was forced to take the value of the overall minimum of the attenuated HRTF magnitudes.

\subsubsection{Microphone array directivity pattern}

If microphone capsules and mounting would be perfect, the theoretical directivity pattern of each microphone on the sphere could be directly used in the attenuated HRTF pattern fitting procedure. However, due to deviations in microphone placement, level and phase differences between microphones, and influences of windshield, cable outlet and fixation mechanisms, the real directivity pattern will deviate from the theoretical one. Therefore, the real directivity pattern of each microphone on the sphere has been characterized at 614 directions with a dedicated measurement setup in an anechoic room.

In order to enable a continuous representation and to smooth out pattern irregularities, a spherical harmonic decomposition has been conducted on the magnitude and spectrally unwrapped phase, in a similar way as on the target directivity pattern. Here, the same order $N=18$ has been used, with a Tikhonov regularization that favors lower orders. For frequencies lower than $200 \mathrm{~Hz}$, only the spherical harmonic coefficients until $N=3$ are taken into account to eliminate fast varying spatial fluctuations from loudspeaker instabilities and phase noise. Indeed, at these frequencies, the size of the sphere is lower than $1 / 10^{\text {th }}$ of the wavelength, and spatial variations will be rather small.

\subsubsection{HRTF directivity pattern fitting}

Based on the characterization of the target directivity pattern and the directivity pattern of each element of the array in terms of spherical harmonics, the appropriate filter coefficients $W_{i}(f)$ can be calculated so that the resulting array directivity pattern approaches the attenuated HRTF directivity pattern:

$$
\operatorname{attHRTF}(f, \phi, \theta)=\sum_{i=1}^{32} W_{i}(f) \operatorname{MIC}_{i}(f, \phi, \theta),
$$

where attHRTF $(f, \phi, \theta)$ and $\operatorname{MIC}_{i}(f, \phi, \theta)$ are the complex-valued directivity pattern of the attenuated HRTF and $i$ th microphone on the sphere. Different techniques have been developed to find the frequency dependent weights $[27,28]$. In this work, we will estimate the microphone weights $W_{i}(f)$ from a regularized least-square solution of the discretized version of Eq. (4):

$$
\mathbf{W}(f)=\left[\operatorname{MIC}^{H}(f) \operatorname{MIC}(f)+\lambda(f) \mathbb{I}_{32}\right]^{-1} \operatorname{MIC}^{H} \operatorname{attHRTF}(f) .
$$

The matrices attHRTF $(f)$ and MIC $(f)$, respectively of size $M \times 1$ and size $M \times 32$, contain the complex-valued smoothed directivity patterns of the target attenuated HRTF and measured microphone patterns, found by evaluating the magnitude and phase spherical harmonic resynthesis (Eq. (2)) at each direction $\left(\phi_{m}, \theta_{m}\right)$ of a uniformly sampled $M$-point evaluation grid, allowing for flexibility regarding head orientation and overall accuracy. The regularization term $\lambda(f) \mathbb{I}_{32}$ in Eq. (5) is used to avoid over-fitting and to decrease the sensitivity of the fit to sensor noise and errors in e.g. microphone characteristics. More complex expressions for the regularization term exist, e.g. with direct inclusion of the desired white noise gain (WNG) in the regularization term [28, 29].

\subsection{Auralization based on a 2-channel spherical head approximation}

The pattern emulation technique shows good results at frequencies below a given cross-over frequency $f_{X}$, typically around $2 \mathrm{kHz}$ (cf. Section 3.2 and [30, 31]). At higher frequencies, the attenuated HRTF pattern fitting performance decreases steadily due to inaccuracies in the target and measured directivity patterns and spatial aliasing caused by the microphone spacing, which is no longer small compared to the corresponding wavelength at these frequencies. In order to provide the listener with a full-spectrum binaural signal, the pattern emulation technique is complemented with a 2-channel spherical head approximation, by taking the signal from the microphone closest to the location of the ears [7]. As directional dependent noise mitigation with the angular IL is no longer possible in this case, a more basic approach is followed: the signal is attenuated with the overall IL of the complete left or right frontal hemisphere. For sources in the corresponding hemisphere, the attenuation will be reasonably correct, but sources in the opposing hemisphere will be subject to the same attenuation levels as well. However, it should be noted that sources in the contralateral hemisphere will have relatively less influence, as they are shielded by the hard sphere. 


\section{Auralization of the effect of an L-shaped mound near a highway}

The realism of the proposed auralization methodology has been evaluated in a case-study, by considering the effect of a complex L-shaped mound on the soundfield. The mound is located parallel to the E17 highway near Kruishoutem (Belgium) and has dimensions $84.5 \mathrm{~m} \times 65.3 \mathrm{~m}$ with an average height of $6 \mathrm{~m}$. Before construction of the mound, a dense laurel hedge was present, following the L-shape of the mound. The highway is located on an embankment of about $1.7 \mathrm{~m}$ high. The distance between the center of the first lane and the top center of the mound is estimated at $27 \mathrm{~m}$. Directly behind the mound is an open workshop, constructed with lath partitioning walls.

Different positions of interest were chosen at the site. MIC1 is chosen just in front of the mound, serving as a reference position to be able to detect changes in the source level and spectra. To measure the effect of the mound, MIC2 is located behind the (future) mound (cf. Fig. 3).

The perceived realism of the auralization mainly depends on the accuracy of the simulated (angular) IL. Therefore, in a first step, the noise reduction of the mound will be expressed quantitatively, based on simulations of the insertion loss with three different models: ISO 9613-2 [32], Pierce's barrier diffraction model [33] and the Harmonoise model [34]. ISO 9613-2 [32] is a general outdoor sound propagation engineering model, consisting of an empirical screening formula. The mound is approached by two thin screens, positioned at each of the mound's horizontal diffraction edges. The Pierce barrier diffraction model [33] is a more elaborated model, where the mound is modeled as a rigid three-sided semi-infinite barrier in terms of auxiliary Fresnel functions. In the Harmonoise model [34], calculation of the diffraction around each horizontal edge is based on a numerical approximation of the diffraction integrals in function of the Fresnel number. This model allows for the inclusion of the full geometry of the measurement site, while the acoustical impedances of the mound and various ground surfaces can be explicitly taken into account in terms of effective flow resistivities. The accuracy of the proposed simulation techniques will be put to the test by comparing simulated ILs with the measured IL, based on measurements before and after installation of the mound. In a second step, the effect of the mound on the sound environment is auralized, based on the IL calculated with the aforementioned propagation models and array recordings taken before the mound was installed. To investigate if the designed 32-channel microphone array is suitable for the proposed auralization technique, the accuracy of the attenuated HRTF pattern emulation is evaluated in terms of relative mean square error (rMSE) and white noise gain (WNG) (Section 3.2). Furthermore, the perceived realism is evaluated by listening tests (Section 3.3), in which auralized samples are compared to real-life recordings after the mound was erected.

\subsection{Modeling the highway IL of the mound}

Based on the pass-by of a single source, Salomons [35] formulated an expression for the $L_{\mathrm{eq}, \mathrm{lh}}$ of a constant flow of sources along a single line (incoherent line source), measured at a sufficiently large distance $d$ from the line source:

$$
L_{\mathrm{eq}, 1 \mathrm{~h}}=10 \log _{10}\left(\sum_{j} 10^{\left(L_{W}\left(\phi_{j}\right)-10 \log _{10}(4 \pi d)-A_{\mathrm{ex}}\left(\phi_{j}\right)\right) / 10} \delta \phi_{j}\right)+10 \log _{10}\left(\frac{Q}{v}\right) .
$$

Here, the source model is based on the segmentation of the line source into different angular sectors, with size $\delta \phi_{j}$ (in radians), each represented by a single point source at the center (cf. Fig. 3). In Eq. (6), $L_{W}\left(\phi_{j}\right)$ represents the sound power level of the point source, representative for each vehicle category, modeled following the Harmonoise/Imagine source model [36]. $A_{\mathrm{ex}}\left(\phi_{j}\right)$ is the excess attenuation, calculated using the three aforementioned models for a $2 \mathrm{D}$ cross-section at angle $\phi_{j}$ as the difference between the level at the receiver in free field and the level at the receiver in the real situation. $A_{\mathrm{ex}}\left(\phi_{j}\right)$ includes the air absorption calculated with ISO 9613-1 [37], ground interaction (based on an extended version of ISO 9613-2 [38], except for the Harmonoise model) and attenuation by vegetation present prior to the installation of the mound or mound attenuation and turbulent scattering after installation of the mound. The attenuation of the vegetation (laurel hedge) is estimated by using a semi-empirical model developed by Aylor [39], with an effective Leaf Area Density of $4 \mathrm{~m}^{-1}$ and leaf width of $10 \mathrm{~cm}$, experimentally validated by Van Renterghem et al. [40]. Turbulent scattering is included based on a model developed by Forssén et al. [41]. The turbulence strength parameters $C_{v}^{2}=1.0095 \mathrm{~m}^{4 / 3} / \mathrm{s}^{2}$ and $C_{T}^{2}=0 \mathrm{~K}^{2} / \mathrm{m}^{2 / 3}$ are estimated based on the measured meteorological conditions $[42,43]$. For the application of the Harmonoise propagation model, the effective flow resistivities of the different surfaces were chosen based on visual inspection of the ground and tabulated values. For the ISO 9613-2 simulation 
model, $G$ is estimated as $G=0.1$ near the source (hard ground) and $G=0.6$ (medium porosity) for ground surfaces in the middle region and at the receiver. The last term in Eq. (6) includes properties of the traffic flow: $Q$ is the average number of sources passing per hour and $v$ is the speed in $\mathrm{m} / \mathrm{h}$.

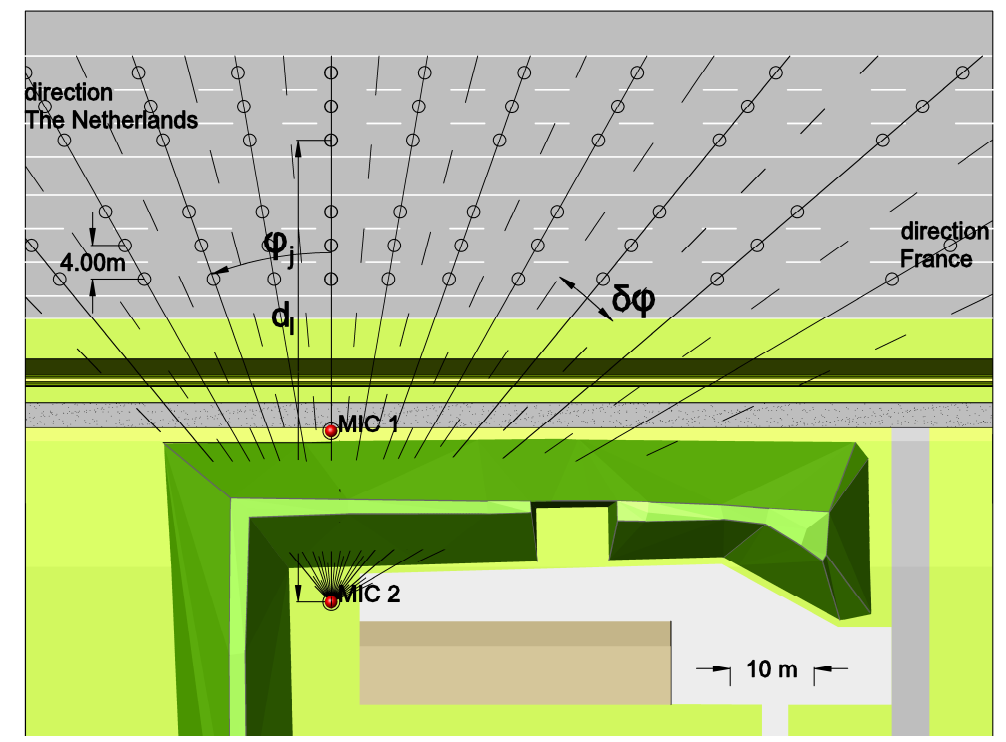

Figure 3: Top view of the mound near the E17 highway. Locations of the measurement microphones are indicated as MIC1 and MIC2. The dashed lines indicate the segmentation boundaries of the different incoherent line sources, based on a constant angular sector $\delta \phi$. The equivalent point sources are illustrated by the circles at the centers of each segment. $L_{\mathrm{eq}, 1 \mathrm{~h}}\left(\phi_{j}\right)$ is calculated along each $2 \mathrm{D}$ source-receiver intersection, with angle $\phi_{j}$ relative to the normal to the highway. For illustrative purposes $\delta \phi=5^{\circ}$, for the simulations $\delta \phi=2^{\circ}$ was used.

If the distance between receiver and line source is large enough, the angular segmentation can be kept constant [35]. This constant angular segmentation results in a fine segmentation for sources close to the receiver, which should be modeled with more care as they contribute most. For large distances (at large angles) relative contribution to the receiver is smaller and the length of the segments is proportionally increased. In the following simulations, an angular sector of $2^{\circ}$ will be used.

For the calculation of the noise immission at a receiver, the contribution of different line sources, representing different vehicle categories on a multitude of lanes (six in this case), has to be taken into account. Here, we are not only interested in the total sound immission at a given receiver point before and after installation of the mound (total $L_{\text {eq,NM }}$ and $L_{\text {eq,M }}$ ), but also in the angular levels originating from a given direction $\phi_{j}$. Therefore, the sound energy of each source from the same angular sector $\phi_{j}$ (cf. Fig. 3), corresponding to different lanes and different vehicle categories, is summed:

$$
L_{\text {eq, } 1 \mathrm{~h}}\left(\phi_{j}\right)=10 \log _{10}\left(\sum_{l=1}^{6} \sum_{\text {cat }=1,3} \sum_{h=0.01,0.03 \mid 0.75} \frac{Q_{l, \text { cat }}}{v_{\text {cat }}} 10^{\left(L_{W, \text { cat }, h}\left(\phi_{j}\right)\right) / 10} \times 10^{\left(-10 \log _{10}\left(4 \pi d_{l}\right)-A_{\text {ex }, l, h}\left(\phi_{j}\right)\right) / 10} \delta \phi\right) .
$$

Eq. 7 incorporates a summation over 20 sources: two sources at heights $h=0.01 \mathrm{~m}$ and $h=0.3 \mathrm{~m}$ representing category 1 vehicles on each of the six lanes and two sources at heights $h=0.01 \mathrm{~m}$ and $h=0.75 \mathrm{~m}$ representing category 3 vehicles on lane 1, 2, 5 and 6. No heavy traffic is assumed on the left lanes (lanes 3 and 4), while vehicles of category 2 are not included, as no data was available. As not all sources equally contribute to the angular level, each source is weighted with the average number of vehicles per unit length $\left(Q_{l \text {,cat }} / v_{\text {cat }}\right)$, with $v_{1}=120 \mathrm{~km} / \mathrm{h}$ and $v_{3}=90 \mathrm{~km} / \mathrm{h}$. From traffic counts, the average number of vehicles in category 1 and 3 in each direction of the highway is known [44]. Based on these average values, an estimate is made for the distribution of vehicles over the three lanes in each direction: the light vehicles are distributed according to 20\% (right lane), 60\% (middle lane), 20\% (left lane), while the 
heavy traffic is distributed as $85 \%, 15 \%$ and $0 \%$.

With Eq. (7), the sound immission from the highway, originating from a given direction $\phi_{j}$, can be calculated without and with the mound $\left(L_{\mathrm{eq}, \mathrm{NM}}\left(\phi_{j}\right)\right.$ and $\left.L_{\mathrm{eq}, \mathrm{M}}\left(\phi_{j}\right)\right)$. The angular $\operatorname{IL}\left(\operatorname{IL}\left(\phi_{j}\right)\right)$ is defined as the difference between both.

In a similar way, the total IL is the difference between the total level before and after installation of the mound.

\subsubsection{Propagation model validation}

To evaluate the accuracy of the different propagation models, results are compared with field measurements. In this study, the total IL will be used as an indicator of the expected model accuracy. For a more extensive validation of the angular IL, the reader is referred to previous work [30]. In order to measure the total highway IL of the mound, long-term level measurements have been performed with class 1 measurement equipment at MIC1 and MIC2 (cf. Fig. 3) before and after construction of the mound. $L_{\mathrm{eq}, 1 \mathrm{~h}}$ was continuously logged from June $1^{\text {st }} 2012$ until July $6^{\text {th }}$ 2012 and from October $16^{\text {th }} 2012$ until November $23^{\text {th }} 2012$ for the case without and with the mound. For the analysis, only $L_{\mathrm{eq}, \mathrm{h}}$-values for periods with weak wind $(v<6 \mathrm{~m} / \mathrm{s})$ and no rain $(<0.05 \mathrm{~mm}, \mathrm{RH}<95 \%)$ are retained. Furthermore, when precipitation was measured, measurements of that hour and the following two hours were discarded to avoid wet microphone windscreen influence [45].

Based on the remaining measurements, an estimate for the highway IL of the mound at MIC2 is calculated by subtracting the transmission loss between MIC1 and MIC2 before and after construction of the mound.

Fig. 4 shows a comparison of the measured IL with the simulated IL. It can be seen that the Harmonoise model predicts the measured highway IL of the mound most accurately. The IL deviates less than $3.5 \mathrm{~dB}$ at frequencies below $10 \mathrm{kHz}$. At low frequencies, Pierce's model underestimates the IL, while at mid frequencies an overestimate of about $5 \mathrm{~dB}$ is made.

The IL based on the ISO 9613-2 model shows the largest deviations from the measured IL. At mid frequencies, the simulated IL reaches values of $16 \mathrm{~dB}$, an overestimate of almost $10 \mathrm{~dB}$. Furthermore, almost no correspondence with the spectral shape of the IL is seen.

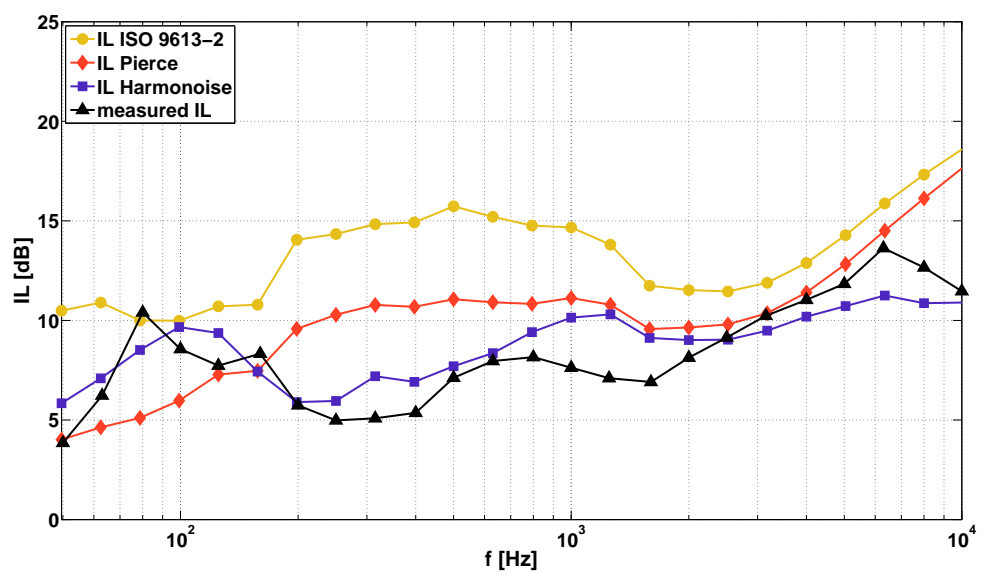

Figure 4: Comparison of the measured IL at MIC2 with the calculated IL based on the ISO 9613-2 model (circle), Pierce's model (diamond) and the Harmonoise model (square).

\subsection{Evaluation of the pattern emulation accuracy}

Using the methodology described in Section 2, the effect of the mound on the sound environment is obtained by subtracting the calculated angle dependent IL from the HRTF for each corresponding direction. Here, it is assumed that the angular IL is independent of elevation, since this dependency cannot be simulated by the models that have 
been used. However, as the human hearing has only limited capabilities to estimate the elevation of the source, this is not expected to have a noticeable influence.

The decomposition into spherical harmonics assures a continuous representation, by leveling out the discrete jump at $\phi= \pm 85^{\circ}$, arising from the fact that the angular $\operatorname{IL}(\phi)$ of the mound has non-zero values for $-84^{\circ} \leq \phi \leq 84^{\circ}$ and zero values at $86^{\circ} \leq \phi \leq 274^{\circ}$. For the determination of the frequency dependent microphone weights $W_{i}(f)$, a uniform grid with $M=614$ unique points is chosen.

The accuracy of the pattern fit that can be obtained with the 32-channel microphone array can be expressed in terms of relative mean square error (rMSE), given by Eq. (8), and white noise gain (WNG), given by Eq. (9):

$$
\begin{aligned}
\operatorname{rMSE}(f) & =10 \log _{10} \frac{\sum_{m=1}^{M} v_{m}\left|\widehat{\operatorname{attHRT}} \mathbf{F}\left(f, \phi_{m}, \theta_{m}\right)-\operatorname{attHRTF}\left(f, \phi_{m}, \theta_{m}\right)\right|^{2}}{\sum_{m=1}^{M} v_{m}\left|\operatorname{attHRTF}\left(f, \phi_{m}, \theta_{m}\right)\right|^{2}}, \\
\operatorname{WNG}(f) & =10 \log _{10} \frac{\mathbf{W}^{H}(f) \mathbf{M I C}^{H}(f) \operatorname{diag}\left(v_{1} \ldots v_{M}\right) \mathbf{M I C}(f) \mathbf{W}(f)}{\mathbf{W}^{H}(f) \mathbf{W}(f)}
\end{aligned}
$$

with $\operatorname{attHRTF}\left(f, \phi_{m}, \theta_{m}\right)$ representing the target directivity pattern (after smoothing), attHRTF $\left(f, \phi_{m}, \theta_{m}\right)$ the emulated directivity pattern obtained with the 32-channel microphone array, and $v_{m}$ the Voronoi weights.

The rMSE indicates the accuracy of the pattern fit by calculating the relative error between the target and emulated directivity pattern. The WNG, defined according to Rasumow et al. [46], evaluates the dependency of the accuracy on small errors in microphone characteristics, robustness of the resulting filter coefficients and the sensitivity to microphone noise. The WNG is defined by relating the mean array gain, with inclusion of the microphone directivity patterns, to the array gain for spatially uncorrelated noise as input for each channel.

Generally, the rMSE improves when decreasing the regularization parameter $\lambda$, while the WNG deteriorates. Ideally, $\lambda$ should be chosen to maximize the WNG in order to decrease the effect of microphone noise [29]. Given the noise level of the selected microphones (estimated at $35 \mathrm{~dB}(\mathrm{~A})$ on average [11]), a value of $\lambda=1$ would be advisable to yield perceptually good results for recordings in quiet environments [29]. However, as this microphone array is to be used for the auralization of the effect of noise mitigation measures on traffic in already noisy environments (a background noise level of approximately $60 \mathrm{~dB}(\mathrm{~A})$ was observed), the WNG constraint can be relaxed and more effort can be put into improving the accuracy of the pattern fit (rMSE), allowing for lower $\lambda$. Here, $\lambda=0.01$ is chosen.

The accuracy of the pattern emulation technique with the designed 32-channel microphone array is given in Fig. 5. The rMSE ranges between $-20 \mathrm{~dB}$ at the lowest frequencies and $-10 \mathrm{~dB}$ at $1 \mathrm{kHz}$. The rMSE reaches $-5 \mathrm{~dB}$ at $2 \mathrm{kHz}$, and approaches $0 \mathrm{~dB}$ at higher frequencies.

The WNG ranges between $-30 \mathrm{~dB}$ at low frequencies and $-20 \mathrm{~dB}$ at $1 \mathrm{kHz}$, while WNG levels fluctuate around $-10 \mathrm{~dB}$ at higher frequencies.

It can be seen that the rMSE and WNG both depend on the target directivity pattern, and more specific, the used propagation model. At mid frequencies, the pattern fit to the HRTF attenuated with the Harmonoise propagation model shows the best performance regarding both rMSE and WNG. The fit with the Pierce model performs slightly worse, while the fit with the ISO 9613-2 model performs the least. The difference in performance between the fit with the Harmonoise model and ISO 9613-2 model is largest near $250 \mathrm{~Hz}$ (3.5 dB for the rMSE and $5 \mathrm{~dB}$ for the WNG), but decreases when the frequency rises.

\subsection{Evaluation of the realism of the auralization methodology}

The proposed methodology for predicting the perceived effect of a noise mitigation measure based on a priori recordings is considered valid if the effect of the measure can clearly be noticed by the average listener and this listener cannot distinguish between the auralization based on a priori recordings and a posteriori recordings. To test whether this is the case, two forced choice experiments are conducted. The first experiment forces participants to decide whether a sound presented to them binaurally was obtained before or after the noise mitigation measure was installed. The auralization methodology passes the test if auralizations and a posteriori recordings are both assigned to the category representing the situation with the noise mitigation measure in place. The second experiment forces 


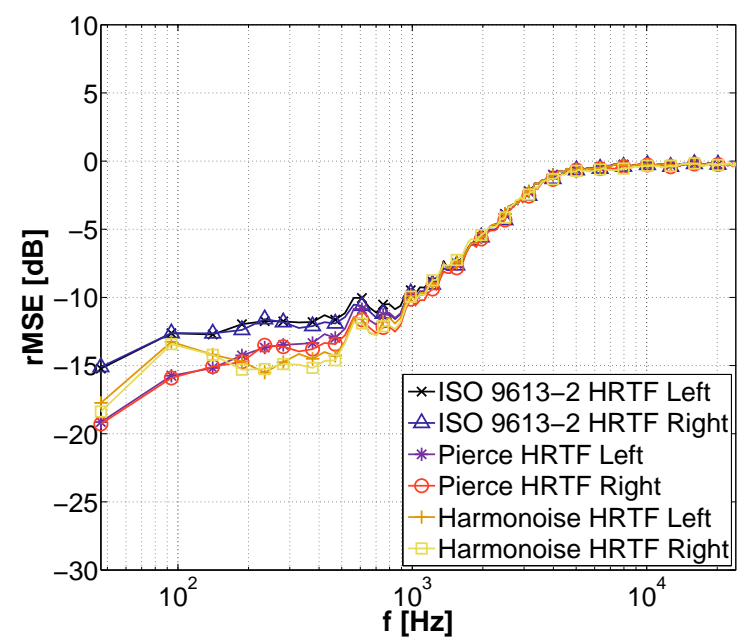

(a)

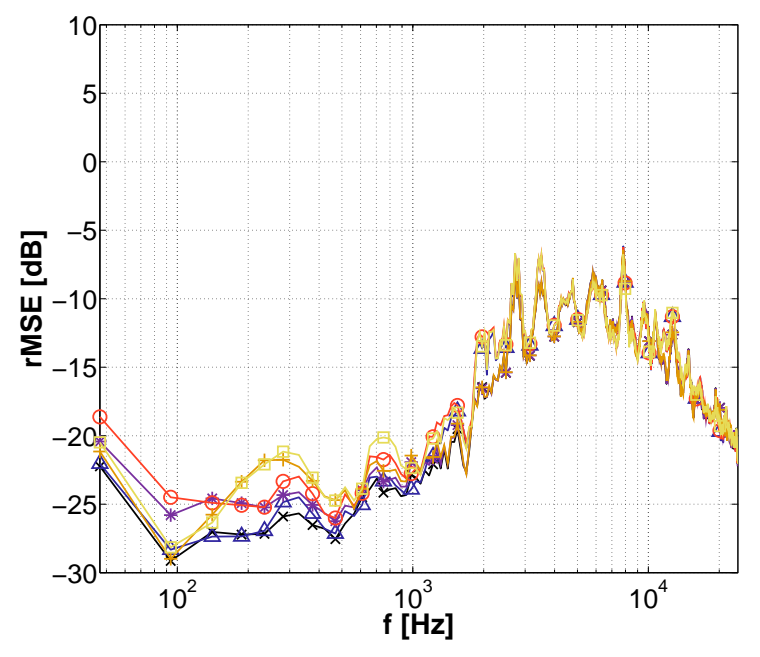

(b)

Figure 5: Comparison of the attenuated HRTF pattern fitting accuracy with the different propagation models: (a) rMSE and (b) WNG. The fitting has been performed with a uniformly sampled grid (614 unique points, $\lambda=0.01)$.

the listener to decide whether a sound that is presented to them is an auralization or an a posteriori recording. In forced choice experiments where the participant cannot extract any useful information to support his or her decision, both categories are equally often chosen. Hence the test passes if both the auralizations and the a posteriori recordings end up equally often in both categories. This second experiment is somewhat refined by including different variants of auralizations. By testing whether one of the variants ends up in the category judged to be a prediction statistically more significantly, it can be concluded that this imitation of reality fails, and, depending on the statistical significance, to what extent.

\subsubsection{Audio sample acquisition and auralization}

Recordings before and after installation of the mound have been made with the 32-channel microphone array as required by the methodology presented in Section 2. For comparison, a generally accepted HATS binaural recording using a B\&K Type $4128 \mathrm{C}$ with inner ear simulator Type $4158 \mathrm{C}$ is used. All recordings are made at the MIC2 position.

In the a priori case, 17 binaural samples have been extracted from the microphone array recordings and the corresponding samples from the HATS recording have been selected as well. After installation of the mound, 17 samples have been extracted from the array recordings and 11 samples from the HATS recordings. The latter will be used as reference samples to evaluate the performance of the auralization technique. To allow a proper evaluation, samples were close to $30 \mathrm{~s}$ long and contained several distinct truck passages. Some samples also contained other sounds, not related to traffic (e.g. a barking dog).

To create binaural extractions, corresponding with the orientation of the HATS, the array recordings are first filtered with a low-pass and high-pass filter with cross-over frequency $f_{X}=1782 \mathrm{~Hz}$, determined from earlier research [30, 31]. Each of the low-pass filtered microphone signals is filtered with the array coefficients, either calculated to include the effect of the mound (to create an auralization based on a priori recordings) or without effect of the mound (to create a binaural extraction from array recordings) by setting the angular IL in Eq. (1) to zero (see Section 2.2). At frequencies higher than $1782 \mathrm{~Hz}$, only the high-pass filtered signal from the microphones corresponding to the left and right ear direction is kept and attenuated with the hemispherical IL to simulate the effect of the mound or left unchanged in case of an a posteriori recording (see Section 2.3). Given the fixed orientation of the reference HATS recordings, the microphone array weights as well as the microphones for the left and right ear were selected corresponding to the orientation of the HATS and remained fixed in time.

In this paper, an additional auralization has been created as well, by using the 2-channel spherical head approximation with hemispherical IL over the full frequency range (Section 2.3). 


\subsubsection{Listening test set-up and results}

Different tests have been designed to evaluate the realism of the auralized effect of the mound, either by investigating the plausibility (if the listener evaluates samples by referring to his/her experience or expectation) [47], or authenticity (when an external reference is explicitly given) [48] of the auralized sample. The listening test was performed with a calibrated headphone (Sennheiser HD-280 pro) and took place in the listening room at Ghent University or in a quiet room at the listener's home. No head-tracking was provided, as it was assumed that during the test the focus of the listener was fixed towards the computer screen, corresponding with the fixed orientation of the reference HATS recording. In total, 14 female listeners and 23 male listeners with normal binaural hearing (tested with pure tone audiometry) and ages ranging between 21 and 57 years participated in the tests. 10 listeners originated from the acoustics research group, while the others had little to no acoustical background.

In a first test, it is checked whether or not listeners can distinguish between recordings made before installation of the mound and recordings made after installation. HATS recordings, binaural samples from array recordings without the mound present and some auralized samples including the simulated effect of the mound are included. In a forced choice test, listeners were asked to indicate whether the sound fragments were recorded before or after construction of the mound.

The results of this test are given in Table 1. The rightmost column gives the probability that the null-hypothesis, assuming random choice, is accepted, resulting from a two-sided binomial test.

The results of this test clearly indicate that listeners can indeed distinguish between fragments recorded before and after construction of the mound, whether or not fragments have been binaurally synthesized from a microphone array recording, recorded with a HATS, or synthesized based on a priori recordings using the proposed methodology. Furthermore, the attenuation model used for implementing the proposed auralization technique had no effect.

\begin{tabular}{lcccc}
\hline \hline Samples presented to listener & Indicated before & Indicated after & Count & p-value \\
\hline HATS recording before & 74 & 0 & 74 & $<0.01$ \\
Array recording before & 73 & 1 & 74 & $<0.01$ \\
HATS recording after & 5 & 32 & 37 & $<0.01$ \\
Array recording after & 1 & 36 & 37 & $<0.01$ \\
Array auralization (Harmonoise) & 1 & 36 & 37 & $<0.01$ \\
Array auralization (Pierce) & 0 & 37 & 37 & $<0.01$ \\
Array auralization (ISO 9613-2) & 0 & 37 & 37 & $<0.01$ \\
\hline \hline
\end{tabular}

Table 1: Results of the first test. The columns indicate the responses given by the listeners, presented with auralized samples and samples recorded before or after installation of the mound. The right column gives the probability that the null-hypothesis, assuming random choice, is accepted. $\left(\chi^{2}=303.81, p<0.01\right)$

In a second test, the plausibility of the auralized samples was investigated, again by using a forced choice setup. Therefore, the listener is presented with ten audio fragments and he or she was asked to indicate if the given sample was a true recording or an auralization. The samples presented to each participant contained four HATS recordings made after installation of the mound and six samples auralised using the proposed technique, presented in Section 2, using the different propagation models. Out of these six samples, three samples were created by using the 2-channel spherical head approximation with hemispherical IL (Section 2.3) for the full frequency range ('L-R auralization'). Table 2 indicates whether listeners categorize the fragment as a recording or a simulation. The data in this table shows that listeners cannot find any clues in the sound samples to support their decision and hence make a random choice. Only for the samples auralized using the full range 2-channel spherical head approximation combined with the ISO 9613-2 propagation model the assumption of random choice was rejected, as almost $73 \%$ of the samples were indicated to be simulated. For auralizations based on array pattern emulation with the ISO 9613-2 model attenuated pattern, an increased number of samples $(65 \%)$ have been identified as simulations. However, the assumption of random choice cannot be rejected with $99 \%$ certainty. 


\begin{tabular}{lcccc}
\hline \hline Samples presented to listener & Indicated recording & Indicated simulation & Count & p-value \\
\hline HATS recording after & 84 & 64 & 148 & 0.118 \\
Array auralization (Harmonoise) & 15 & 22 & 37 & 0.324 \\
Array auralization (Pierce) & 19 & 18 & 37 & 1 \\
Array auralization (ISO 9613-2) & 13 & 24 & 37 & 0.099 \\
L-R auralization (Harmonoise) & 19 & 18 & 37 & 1 \\
L-R auralization (Pierce) & 23 & 14 & 37 & 0.188 \\
L-R auralization (ISO 9613-2) & 10 & 27 & 37 & $<0.01$ \\
\hline \hline
\end{tabular}

Table 2: Results of the second test. The columns indicate the responses given by the listeners, presented with auralized samples and samples recorded after installation of the mound. The right column gives the probability that the null-hypothesis, assuming random choice, is accepted. $\left(\chi^{2}=17.31, p<0.01\right)$

In order to further investigate the authenticity of the auralized samples and check whether or not a difference in similarity between an a posteriori recording and the different auralization methods can be heard, a similarity ranking test is performed. In this test, the listener is presented with a reference sample, recorded after installation of the mound, and six auralizations. Three auralizations are made with the array pattern emulation technique (Section 2.2) with each of the three different propagation models, while the other three are created with the full range 2-channel spherical head approximation technique with inclusion of the hemispherical IL (Section 2.3). The auralized samples all originate from the same array recording made before installation of the mound, and as such have the same content in terms of car and truck passages, but this content is different from the content of the a posteriori reference sample. The listener is then asked to rank the simulated samples according to their similarity with the sound environment after installation of the mound, represented by the HATS recording, given as a reference. It is pointed out that the reference and auralized samples have different content.

Table 3 shows the frequency count of rankings given to each of the auralization methodologies. From this table, it can be seen that the full range 2-channel spherical head approximation technique combined with the ISO 96132 propagation model receives the worst ranking. Indeed, after the test, listeners indicated that these samples were somewhat easier to detect, due to the large difference between the left and right ear level. It is more difficult to draw conclusions regarding the other techniques.

In order to compare the different techniques and investigate if the mean ranking of the samples is significantly different, a multiple comparison test is performed by applying Tukey's least significant difference procedure on the outcome of an ANOVA test. Fig. 6 shows the mean ranking, together with the 95\% confidence intervals. The array emulation technique with Pierce's model performs best based on absolute ranking, although no significant difference is seen for the pattern emulation techniques with other propagation models. However, this technique is significantly better than the full range 2-channel spherical head approximation technique combined with the Harmonoise and ISO 9613-2 propagation model. Typically, the pattern emulation technique outperforms the full range 2-channel spherical head approximation technique with a given propagation model, although the difference is only marginally significant.

\begin{tabular}{lcccccc}
\hline \hline Auralization technique & 1 & 2 & 3 & 4 & 5 & 6 \\
\hline Array auralization (Harmonoise) & 8 & 4 & 11 & 8 & 5 & 1 \\
L-R auralization (Harmonoise) & 6 & 5 & 3 & 8 & 11 & 4 \\
Array auralization (Pierce) & 8 & 10 & 9 & 5 & 3 & 2 \\
L-R auralization (Pierce) & 6 & 5 & 6 & 11 & 8 & 1 \\
Array auralization (ISO 9613-2) & 7 & 13 & 5 & 2 & 6 & 4 \\
L-R auralization (ISO 9613-2) & 2 & 0 & 3 & 3 & 4 & 25 \\
\hline \hline
\end{tabular}

Table 3: Influence of the auralization technique. The columns indicate the ranking given by the listeners, presented with samples generated by different auralization techniques ( 1 means most similar). The total sample count is 37 . $\left(\chi^{2}=116.92, p<0.01\right)$ 


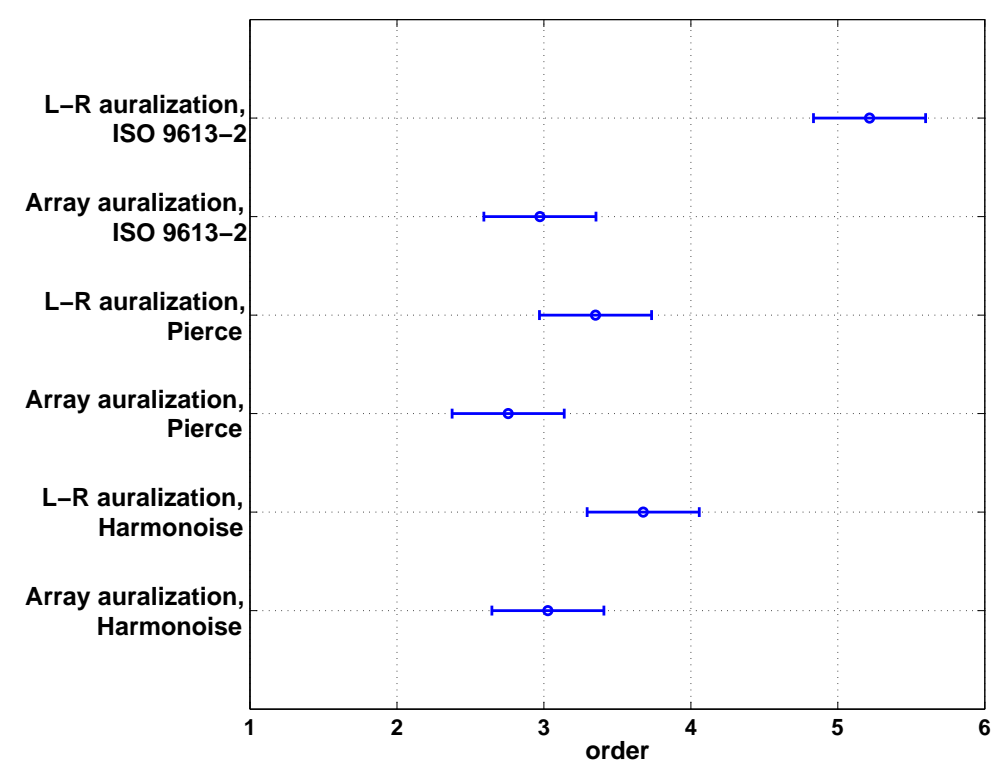

Figure 6: Results of the subjective ranking of the different auralization techniques (cf. Table 3). Mean ranking and 95\% confidence intervals of each technique are indicated (1 means most similar).

\section{Conclusion}

A new methodology has been introduced to evaluate the effect of a noise mitigation measure in a complex sound environment by means of a priori microphone array recordings and simulations of the angular IL. In this methodology, an auralization of the predicted sound environment after introduction of the noise mitigation measure is obtained by weighting the array signals so that the resulting directivity pattern approximates the left and right ear HRTF, attenuated with the insertion loss of the mitigation measure for each direction. At higher frequencies, where spatial sampling on the spherical microphone array is no longer sufficiently dense, the signal of the microphone on the hard sphere closest to the left and right ear position, attenuated by the hemispherical IL, is used instead.

The validity of this technique has been confirmed in a case-study, where it has been investigated to what extent the effect of a large L-shaped mound on the sound environment can be auralized.

Three different techniques have been used to estimate the angular IL of the mound: the ISO 9613-2 propagation model, the Pierce barrier diffraction model and the Harmonoise point-to-point model. The physical accuracy of these engineering models has been put to the test. It was found that the measured IL was best approximated by the IL calculated with the Harmonoise model, which accounted best for spectral fluctuations. The IL calculated with the ISO 9613-2 model showed the worst performance, with an overestimate of almost $10 \mathrm{~dB}$ at mid frequencies.

Based on the calculated angular IL and recordings made before the installation of the mound, an auditive preview of the effect of the mound has been created. Both the effect of the introduction of the mound and the realism of the auralization methodology has been investigated by means of forced choice listening tests. From these tests it was seen that the effect of the mound was clearly heard by listeners and this was equally possible for binaural recordings, synthesized samples based on array recordings and auralizations based on array recordings made before the installation of the mitigation measure. Furthermore, the realism of the auralization methodology was shown, as it was seen that listeners could not distinguish between recordings and auralizations, except for the full range 2-channel spherical head approximation technique combined with the ISO 9613-2 propagation model.

When ranking the different auralization techniques according to their similarity with the a posteriori sound environment, the full range 2-channel spherical head approximation technique combined with the ISO 9613-2 propagation model was ranked worst. The pattern emulation techniques performed best, but no significant differences between different propagation models were seen. 
It is thus concluded that the proposed methodology to simulate the sound environment after the introduction of a noise mitigation measure, based on a priori recordings with a spherical array of 32 microphones, delivers a valid impression of the future sound environment. The specific propagation model used to calculate the insertion loss of the noise mitigation does not seem to influence the plausibility and authenticity of the synthesized binaural sound signal, with the exception of the combination of the ISO 9613-2 propagation model and a couple of microphones placed on a sphere at the location of the ears. In general, all approaches using the 32 channels to produce the binaural signals are slightly better, which also indicates that binaural recordings would not be sufficient as a starting point for the direction based method proposed here. Moreover, one can easily imagine situations where the noise mitigation has a much stronger location dependency than the mound used in the experimental validation and thus a 32 microphone technique is clearly more flexible.

\section{Acknowledgments}

The authors are grateful for the support by the agency for Innovation by Science and Technology (IWT), project number IM 140784.

\section{References}

[1] J. Maillard, J. Jagla, Auralization of non-stationary traffic noise using sample based synthesis - comparison with pass-by recordings, in: Proceedings of the 41st International Congress and Exposition on Noise Control Engineering (Inter-Noise 2012), 2012.

[2] J. Maillard, J. Jagla, Real time auralization of non-stationary traffic noise - quantitative and perceptual validation in an urban street, in: Proceedings of the AIA-DAGA Conference on Acoustics, 2013.

[3] J. Jagla, J. Maillard, N. Martin, Sample-based engine noise synthesis using an enhanced pitch-synchronous overlap-and-add method, Journal of the Acoustical Society of America 132 (2012) 3098-3108.

[4] J. Forssén, T. Kaczmarek, J. Alvarsson, P. Lundén, M. E. Nilsson, Auralization of traffic noise within the LISTEN project - preliminary results for passenger car pass-by, in: Proceedings of the 8th European Conference on Noise Control, 2009.

[5] A. Peplow, J. Forssén, P. Lundén, M. E. Nilsson, Exterior auralization of traffic noise within the LISTEN project, in: Proceedings of Forum Acusticum, 2011, pp. 665-669.

[6] P. Thomas, M. Boes, T. Van Renterghem, D. Botteldooren, M. Hornikx, W. Desmet, M. E. Nilsson, Auralisation of a car pass-by behind a low finite-length vegetated noise barrier, in: Proceedings of the 9th European Conference on Noise Control, 2012, pp. 932-937.

[7] R. Algazi, R. Duda, D. Thompson, Motion-tracked binaural sound, Journal of the Audio Engineering Society 52 (2004) $1142-1156$.

[8] R. Algazi, C. Avendano, R. Duda, Estimation of a spherical-head model from anthropometry, Journal of the Audio Engineering Society 49 (2001) 472-479.

[9] D. Perrott, K. Saberi, Minimum audible angle thresholds for source varying in both elevation and azimuth, Journal of the Acoustical Society of America 87 (1990) 1728-1731.

[10] D. Grantham, B. Hornsby, E. Erpenbeck, Auditory spatial resolution in horizontal, vertical and diagonal planes, Journal of the Acoustical Society of America 114 (2003) 1009-1022.

[11] T. Van Renterghem, P. Thomas, F. Dominguez, S. Dauwe, A. Touhafi, B. Dhoedt, D. Botteldooren, On the ability of consumer electronics microphones for environmental noise monitoring, Journal of Environmental Monitoring 13 (2011) 544-552.

[12] W. G. Gardner, K. D. Martin, HRTF measurements of a KEMAR, Journal of the Acoustical Society of America 97 (1995) 3907.

[13] M. J. Evans, J. Angus, A. Tew, Analyzing head-related transfer function measurements using surface spherical harmonics, Journal of the Acoustical Society of America 104 (1998) 2400-2411.

[14] W. Zhang, R. A. Kennedy, T. Abhayapala, Efficient continuous HRTF model using data independent basis functions: experimental guided approach, IEEE transactions on Audio, Speech and Language processing 17 (2009) 819-829.

[15] M. Pollow, K. Nguyen, O. Warusfel, T. Carpentier, M. Müller-Trapet, M. Vorländer, M. Noisternig, Calculation of head-related transfer functions for arbitrary field points using spherical harmonics decomposition, Acta Acustica united with Acustica 98 (2012) 72-82.

[16] E. H. A. Langendijk, A. W. Bronkhorst, Fidelity of three-dimensional sound reproduction using a virtual auditory display, Journal of the Acoustical Society of America 107 (2000) 528-537.

[17] A. Rodriguez-Morales, Real-time sound processing for interactive auralisation of sound insulation, in: Proceedings of Forum Acusticum, 2011.

[18] D. Kistler, F. Wightman, A model of head-related transfer functions based on principal component analysis and minimum-phase reconstruction, Journal of the Acoustical Society of America 91 (1992) 1637-1647.

[19] J. Chen, K. E. Van Veen, K. E. Hecox, A spatial feature extraction and regularization model for the head-related transfer function, Journal of the Acoustical Society of America 97 (1995) 439-452.

[20] R. Tenenbaum, V. Melo, J. Naranjo, Virtual reality: A new approach to validate computer modeling auralizations by using articulation indexes, in: Virtual Reality, Technologies, Medical Applications and Challenges, Nova Science Publishers, 2015 , pp. 55-71.

[21] Z. Haraszy, D. Ianchis, V. Tiponut, Generation of the head related transfer functions using artificial neural networks, in: Proceedings of the 13th World Scientific and Engineering Academy and Society (WSEAS) International Conference on Circuits, 2009. 
[22] D. Zotkin, R. Duraiswami, N. Gumerov, Regularized HRTF fitting using spherical harmonics, in: Proceedings of the IEEE workshop on Applications of Signal Processing to Audio and Acoustics, 2009.

[23] H. Na, C. Lee, O. Cheong, Voronoi diagrams on the sphere, Computational Geometry 23 (2002) $183-194$.

[24] G. Golub, P. Hansen, D. O’Leary, Tikhonov regularization and total least squares, SIAM Journal on Matrix Analysis and Applications 21 (1999) 185-194.

[25] R. Duraiswami, D. Zotkin, N. Gumerov, Interpolation and range extrapolation of HRTFs, in: Proceedings of the IEEE International Conference on Acoustics, Speech and Signal Processing (ICASSP), 2004.

[26] J. Ahrens, M. Thomas, I. Tashev, HRTF magnitude modeling using a non-regularized least-squares fit of spherical harmonics coefficients on incomplete data, in: Proceedings of the 2012 conference of the Asia-Pacific Signal and Information Processing Association (APSIPA), 2012.

[27] A. Farina, A. Capra, L. Chiesi, L. Scopece, A spherical microphone array for synthesizing virtual directive microphones in live broadcasting and in post production, in: Proceedings of the 40th International Audio Engineering Society Conference, 2010

[28] E. Rasumow, M. Blau, S. Doclo, M. Hansen, S. Van de Par, D. Püschel, V. Mellert, Least squares versus non-linear cost functions for a virtual artificial head, in: Proceedings of Meetings on Acoustics, 2013.

[29] E. Rasumow, S. Doclo, S. Van De Par, V. Mellert, The impact of the white noise gain (WNG) of a virtual artificial head on the appraisal of binaural sound reproduction, in: Proceedings of the European Acoustics Association (EAA) Joint Symposium on Auralization and Ambisonics, 2014.

[30] P. Thomas, Tools for urban sound quality assessment, Ph.D. thesis, Ghent University (2014).

[31] P. Thomas, T. Van Renterghem, D. Botteldooren, Array-based HRTF pattern emulation for auralization of 3D outdoor sound environments with direction based muffling of sources, in: Proceedings of the 139th Audio Engineering Society Convention, 2015.

[32] ISO 9613-2:1996, acoustics - Attenuation of sound during propagation outdoors - Part 2: General method of calculation, International Organization for Standardization, Geneva, Switzerland (1996).

[33] A. Pierce, Diffraction of sound around corners and over wide barriers, Journal of the Acoustical Society of America 55 (1974) $941-955$.

[34] E. Salomons, D. Van Maercke, J. Defrance, F. De Roo, The Harmonoise sound propagation model, Acta Acustica united with Acustica 97 (2011) 62-74.

[35] E. Salomons, H. Zhou, W. Lohman, Efficient numerical modeling of traffic noise, Journal of the Acoustical Society of America 127 (2010) 796-803.

[36] H. G. Jonasson, Acoustical source modeling of road vehicles, Acta Acustica united with Acustica 93 (2007) $173-184$.

[37] ISO 9613-1:1993, acoustics - Attenuation of sound during propagation outdoors - Part 1: Calculation of the absorption of sound by the atmosphere, International Organization for Standardization, Geneva, Switzerland (1993).

[38] D. S. Cho, J. H. Kim, T. M. Choi, B. H. Kim, D. Manvell, Highway traffic noise prediction using method fully compliant with ISO 9613: comparison with measurements, Applied Acoustics 65 (2004) 883-892.

[39] D. Aylor, Sound transmission through vegetation in relation to leaf area density, leaf width and breath of canopy, Journal of the Acoustical Society of America 51 (1972) 411-414.

[40] T. Van Renterghem, K. Attenborough, M. Maennel, J. Defrance, K. Horoshenkov, J. Kang, I. Bashir, S. Taherzadeh, B. Altreuther, A. Khan, Y. Smyrnova, H. Yang, Measured light vehicle noise reduction by hedges, Applied Acoustics 78 (2014) 19-27.

[41] J. Forssén, M. Hornikx, D. Botteldooren, W. Wei, T. Van Renterghem, M. Ögren, A model of sound scattering by atmospheric turbulence for use in noise mapping calculations, Acta Acustica united with Acustica 100 (2014) 810-815.

[42] J. Defrance, E. Salomons, I. Noordhoek, D. Heimann, B. Plovsing, G. Watts, H. Jonasson, X. Zhang, E. Premat, I. Schmich, F. Aballea, M. Baulac, F. De Roo, Outdoor sound propagation reference model developed in the European Harmonoise project, Acta Acustica united with Acustica 93 (2007) 213-227.

[43] D. Wilson, V. Ostashev, A reexamination of acoustic scattering in the atmosphere using an improved model for the turbulence spectrum, Technical report, New Mexico State University (1998).

[44] Traffic data, yearly average by workday and by hour for 2010, Verkeerscentrum Vlaanderen, Flemish Government, Department of Mobility.

[45] C. Ribeiro, D. Ecotière, P. Cellard, C. Rosin, Uncertainties of the frequency response of wet microphone windscreens, Applied Acoustics 78 (2014) 11-18.

[46] E. Rasumow, M. Blau, M. Hansen, S. Doclo, S. Van De Par, V. Mellert, D. Püschel, Robustness of virtual artificial head topologies with respect to microphone positioning, in: Proceedings of Forum Acusticum, 2011, pp. 397-402.

[47] R. Pellegrini, Quality assessment of auditory virtual environments, in: Proceedings of the International Conference on Auditory Displays (ICAD), 2001.

[48] J. Blauert, Spatial hearing. The psychophysics of human sound localization, MIT Press, Massachusetts, USA, 1997. 